\title{
AJUSTES FONÉTICO-FONOLÓGICOS DEL FONEMA FRICATIVO /S/ EN DIEZ NIÑOS COSTARRICENSES DE 2 A 4 AÑOS
}

\author{
Phonetic-phonological adjustments of the fricative phoneme $/ \mathrm{s} /$ in ten \\ costarican children from 2 to 4 years
}

\author{
Diego Alexander Ugalde Fajardo*1
}

\begin{abstract}
RESUMEN
En este estudio, se realiza un análisis comparativo de los principales ajustes fonético-fonológicos del fonema/s/ que realizan diez niños y niñas costarricenses en edades de 2 a 3 y de 3 a 4 años durante sesiones individuales de habla espontánea. Sin embargo, se argumentará que algunos de los ajustes realizados no tienen necesariamente una motivación fonológica o etaria estricta, sino que también dependen de factores individuales como el desarrollo de la dentición, el refinamiento de las habilidades articulatorias y el proceso de adquisición de la variante estándar o adulta.
\end{abstract}

Palabras clave: fonema fricativo alveolar, ajuste fonético-fonológico, habla infantil, fonética, fonología.

\begin{abstract}
This study presents a comparative analysis of the main phonetic and phonological adjustments of the phoneme /s/, in ten Costarican children ages 2 to 4, during individual interviews of spontaneous speech. The results show that the group of younger children makes some characteristic adjustments of children's speech (like the oclusivization of/s/) more frequently. On the other hand, some allophones of /s/, which are more common on adult speech, are more frequent in the group of older students. However, not only did the adjustments have a phonologic or age motivation, but also they depend on individual factors. Some of these factors are tooth development, refinement of articulatory abilities, and the process of acquisition of the standard Costarican Spanish phonological model.
\end{abstract}

Key Words: fricative alveolar phoneme, phonetic-phonological adjustments, children's speech, phonetics, phonology.

\footnotetext{
${ }^{1}$ Universidad de Costa Rica. Profesor en la Escuela de Filología, Lingüística y Literatura. Costa Rica. Correo electrónico: die_ug21@hotmail.com

Recepción: 10-08-2017. Aceptación: 12-09-2017.
} 


\section{Introducción}

Este estudio tiene como objetivo determinar los principales ajustes fonético-fonológicos del fonema fricativo alveolar sordo/s/ que realizan diez niños costarricenses de 2 a 3 y de 3 a 4 años en sesiones grabadas individuales de habla espontánea, con el fin de establecer si existen diferencias en los tipos y frecuencias de aparición de dichos ajustes entre los dos grupos de niños. El presente trabajo representa un acercamiento inicial sobre la evolución de estos ajustes en los 10 niños mencionados, y no pretende establecer generalizaciones estadísticas absolutas, sino acercarse al fenómeno de forma exploratoria.

La necesidad de realizar un estudio alrededor del proceso de adquisición de este fonema radica en dos motivos principales. En primer lugar, de acuerdo con numerosos investigadores, entre los que se encuentran Bosch (1983), Vivar y León (2009) y Fernández y Cano (2011), los sonidos alveolares (o dentalizados) fricativos como la /s/ están entre los que más tardíamente adquieren los niños en su desarrollo fonológico debido a su difícil producción, por lo que son más susceptibles a sufrir numerosas "alteraciones o ajustes fonéticofonológicos", así llamados por Soto, León y Torres (2011). En segundo lugar, son escasos los estudios en Costa Rica sobre fonética y fonología en procesos de adquisición infantil, por lo que este es un campo aún por explorar y profundizar.

\section{Estudios previos}

En este apartado se presenta un breve panorama de algunas descripciones realizadas sobre el fonema /s/ en el español americano y costarricense, en estudios de Quesada Pacheco (2010)

y (1996), y Calvo (1997). Además, se exponen los estudios de Fernández y Cano (2011) y León y Torres (2010) sobre los ajustes fonético-fonológicos en el habla infantil.

\subsection{El fonema /s/}

El fonema /s/ puede definirse, adaptando el esquema de Carr (1993), con los rasgos: [+obstruyente], [-sonoro], [+continuo], [+anterior], [+coronal] y [+estridente]. Los ajustes 
fonético-fonológicos que se estudiarán, implican, por lo tanto, una modificación en uno o más de estos rasgos.

Quesada Pacheco (2010, p. 73-77) enumera como principales variantes de /s/ para el español de Costa Rica: la fricativa predorsoalveolar sorda [s], y sonora [z]; la fricativa posdental sorda [s], la aspirada (fricativa glotal sorda [h]), la fricativa glotal sorda [?] y la elisión. Quesada Pacheco (1996, p, 548) menciona, entre otros fenómenos, que /s/ se asimila a veces al sonido siguiente en el habla rápida: “[lo'ma:'lindo] lo más lindo” o bien no se percibe del todo: "[la'fjestas] las fiestas".

Asimismo, Calvo (1997) plantea que las variantes más comunes en el habla culta de San José para la /s/ son, en orden de frecuencia, la fricativa dentalizada sorda [s] la predorsoalveolar sorda /s/, la fricativa dental sonora [ð], la fricativa alveolar sonora [z] y la aproximante faríngea sorda [h] (o aspirada). Calvo (1997, p. 242) arguye que /s/ ha dejado de ser predorsoalveolar para "transfonologizarse en una ápico o predorsodental". Para efectos de la presente investigación, se considerarán "normales" o "esperables" para el español de Costa Rica las variantes dentalizada [s] y alveolar [s], que se transcribirán ambas como [s].

\subsection{El fonema $/$ s/ en el habla infantil}

En cuanto a los estudios sobre fonología infantil, Fernández y Cano (2011, p. 57) describen los principales procesos fonológicos que ocurren en el habla infantil en niños castellanohablantes de 2 a 4 años. Metodológicamente, optan por el cotejo de las formas infantiles frente a las adultas, ya que este es el sistema que los niños dominarán a futuro. Sobre la /s/ y los sonidos obstruyentes, alveolares y sonoros, mencionan que "son extraordinariamente susceptibles a experimentar alteraciones", las cuales no siempre tienen una explicación fonológica; es decir, en muchos casos los ajustes fonológicos están determinados por factores individuales como la edad y el desarrollo de la dentición.

Algunos de los procesos fonológicos que realizan los niños del estudio de Fernández y Cano son: posteriorización (cambio de /s/ a [S]), anteriorización (cambio de /s/ a [ $\theta]$ ), oclusivización (usualmente en ataque silábico, por contagio del rasgo oclusivo), sonorización (de /s/ a [z]), u omisión en coda silábica. La omisión de /s/ en posición final absoluta ocurre, según estos autores, mayormente cuando la /s/ no está cumpliendo una 
función gramatical; es decir, es menos frecuente que se omita la -s del plural, por ejemplo. Por su parte, en posición implosiva sí es más frecuente: “ete” en vez de "este"; "etamos” en vez de "estamos", etc.

Soto, León y Torres (2011, p. 73-74), llaman “ajustes fonético-fonológicos” a las realizaciones “que no coincide[n] con el modelo fonológico del español estándar” (p. 70). Argumentan que estos cambios normales se producen porque los niños están en proceso de adquisición y aprendizaje de su lengua materna, y los clasifican según el tipo de ajuste que se realiza: vocálico, cambio de vocal por consonante o viceversa, cambio en la sonoridad, cambio en la zona de articulación (con cercanía o lejanía de zona), cambio de modo de articulación, ajuste de modo y zona (con cercanía o lejanía de zona), ajuste de sonoridad, modo y zona, aféresis (supresión de sonido en posición inicial de palabra), síncopa (eliminación de sonido en posición interior de palabra), apócope (supresión de sonido al final de palabra), prótesis (inserción de sonido en posición inicial de palabra), epéntesis (inserción de sonido en posición interior de palabra), paragoge (inserción al final), metátesis, diptongación y monoptongación.

Por último, el estudio de Vivar y León (2009) sobre el desarrollo fonológico infantil en niños chilenos determinó no solamente que el sonido /s/, junto con otras fricativas y la vibrante, representan los de más tardía adquisición en el habla infantil, sino también que los procesos como la sustitución de /s/ dejan de darse después de los 4 años aproximadamente, y que la omisión disminuye paulatinamente desde los 3 años hasta desaparecer cerca de los 6 años. Esto lleva a establecer un punto de comparación para la presente investigación sobre los ajustes fonéticos realizados por los niños en cuanto al fonema /s/, a partir del cual resulta esperable notar ajustes en las edades del estudio.

\section{Aclaraciones teóricas y metodológicas}

\subsection{Metodología}

El objetivo principal del estudio es comprobar si existen diferencias en cuanto a las variantes de /s/ entre los diez niños de los rangos de edad mencionados, y plantear así posibles tendencias en cuanto a los ajustes fonético fonológicos que realizan. Como ya se mencionó, 
se entiende por "ajuste fonético fonológico", siguiendo a Soto, León y Torres $(2011$, p. 70), a las realizaciones "que no coincide[n] con el modelo fonológico del español estándar". En el caso de /s/, se trata, pues, de realizaciones alofónicas de /s/ en el español estándar de Costa Rica, generadas como resultado del proceso de adquisición y entrenamiento articulatorio de los infantes.

La población del estudio consiste en 10 niños y niñas divididos en dos grupos de cinco niños según la edad: de 2 a menos de 3 años, y de 3 a menos de 4 años. Las grabaciones y transcripciones fueron realizadas en el marco del proyecto "Perfil léxico del niño costarricense de 2 a 4 años. Área Metropolitana, San José”, dirigido por la Dra. Marielos Murillo Rojas. Estas se realizaron en el centro de estudios de cada niño, por sus propias maestras o bien por colaboradoras de ellas, en entrevistas individualizadas que registraban el habla espontánea. Una vez que se realizó la grabación, se llevó a cabo una transcripción utilizando el formato CHAT (Codes for the Human Analysis of Transcripts), mediante el software CLAN (Computarized Language Analysis). Sobre este formato, señala MacWhihnney (2014, p. 14):

[The CHAT format] provides a standardized format for producing computerized transcripts of face-to-face conversational interactions. These interactions may involve children and parents, doctors and patients, or teachers and second-language learners. (...) The system described here is designed for use with both normal and disordered populations. It can be used with learners of all types, including children, secondlanguage learners, and adults recovering from aphasic disorders.

En cuanto al proceso de recolección y análisis de los datos, cabe aclarar que se transcribieron los primeros cinco minutos completos de cada grabación y luego se registraron todas las realizaciones de /s/ por niño. Se realizó la transcripción fonética y fonológica de cada realización de /s/. Posteriormente, esos datos fueron sistematizados en tablas que incluían los tipos de ajuste escuchados, el acento silábico en el que ocurrió el ajuste (sílabas pretónica, tónica o postónica; o bien palabra átona), la posición silábica del ajuste (ataque o coda, ya que el núcleo silábico en español solo puede tener el rasgo [+ silábico] y el fonema estudiado es [- silábico]), así como la opción de indicar si el ajuste ocurrió en posición inicial 
o final de palabra. Con esta información se construyó una tabla para cada uno de los diez niños, y luego se agruparon los datos en dos tablas para cada grupo etario del estudio.

\subsection{Principales ajustes fonético-fonológicos del fonema $/ \mathrm{s} /$}

Los principales ajustes fonético-fonológicos, enlistados a continuación, se basan en dos insumos principales: la teoría revisada y los datos encontrados en las grabaciones. De esta forma, se pudo corroborar o agregar algún ajuste que no hubiera sido registrado con anterioridad, ya que algunos estudios revisados se enfocaban en otras regiones fuera de Costa Rica. A continuación, se presentan únicamente los conceptos e implicaciones fonéticas de dichos cambios. En la sección de análisis, se presentan algunos ejemplos concretos hallados en el corpus.

- Oclusivización: Estos casos tienen en común que la /s/ pierde su rasgo [+ continuo], por lo cual adquiere las características de una consonante oclusiva o africada. Es decir, ocurre un cambio en el modo de articulación. Las posibilidades que se registraron para la oclusivización fueron las siguientes:

○ /s/ $\rightarrow$ [d]: Esto implica una modificación de la /s/ en: [+sonoro], [-continuo], [-estridente].

$\circ \quad / \mathrm{s} / \rightarrow[\mathrm{t}]: \mathrm{La} / \mathrm{s} / \mathrm{se}$ modifica en los siguientes rasgos: [-continuo], [estridente].

$\circ / \mathrm{s} / \mathrm{a} \rightarrow[\mathrm{ts}]: \mathrm{La} / \mathrm{s} /$ adquiere los rasgos de una africada [-continuo], [+emisión retarada].

- Anteriorización: La anteriorización no implica propiamente un cambio en el rasgo de anterioridad; sin embargo, en los estudios revisados que abordan el tema, se suele llamar anteriorización a los cambios hacia consonante dental, por ejemplo. En general, estos tienen en común que se modifica el rasgo [estridente] al valor negativo. No implican un cambio en el modo de articulación, excepto cuando se hace más aproximante, pero sí en el punto de articulación, hacia la zona dental. Las opciones registradas fueron estas: 
$\circ \quad / \mathrm{s} / \rightarrow[\theta]:$ implica un cambio a [-estridente].

$\circ \quad / \mathrm{s} / \rightarrow[ð]:$ implica cambio a [-estridente] [+sonora].

$\circ / \mathrm{s} / \rightarrow$ [ð]: implica cambio [-estridente] [+sonora] [-consonántica] [obstruyente].

- Posteriorización: En la literatura sobre ajustes fonético-fonológicos del habla infantil se llama posteriorización al cambio de /s/ a [S]. Este ajuste implica un cambio en el rasgo [anterior], de positivo en /s/ a negativo en [J]. Ocurre un cambio en el punto de articulación, pues se hace posalveolar.

- Oclusivización y posteriorización: Estos dos cambios simultáneos se representan como: $/ \mathrm{s} / \rightarrow[\mathrm{t}$ ] , y se modifican los siguientes rasgos: [-continuo], [+emisión retardada], [-anterior], [+alta]. Implica, pues, un cambio en el modo, ya que se realiza como africada, y un cambio en el punto, pues se hace posalveolar.

- Sonorización: Se denomina así al cambio de /s/ a [z]; la única modificación que ocurre es en el rasgo [+sonora].

- Omisión: Se representa como el cambio de /s/a $\emptyset$, es decir, cuando se omite el fonema en cuestión, por lo cual todos los rasgos pasan a negativo.

\section{Análisis de la información}

Se registraron, en total, 377 realizaciones del fonema /s/, 194 de las cuales correspondieron al grupo de niños de 2 a 3 años, y 183 al de 3 a 4 . Las Tablas 1 y 2 resumen los ajustes realizados por los diez niños. La Tabla 1 incluye los datos de los niños de 2 a 3 años; el dos, por su parte, a los de 3 a 4 años. Los números 1-10 de las columnas representan el número asignado a cada niño o niña. En el caso de la oclusivización y la anteriorización, que incluyen tres subtipos cada una, se incluye una fila de "total" que suma los resultados de las tres. La Tabla 3 sintetiza de forma comparativa los datos de los dos grupos. 


\section{Tabla 1}

Niños de 2 a 3 - Síntesis global de ajustes realizados

\begin{tabular}{|c|c|c|c|c|c|c|}
\hline \multicolumn{2}{|c|}{ Ajustes } & \multicolumn{5}{|c|}{ Niño(a) } \\
\hline Ajuste & Subtipo & 1 & 2 & 3 & 4 & 5 \\
\hline Normal & & $9,09 \%$ & $50 \%$ & $0 \%$ & $83 \%$ & $35,14 \%$ \\
\hline \multirow{4}{*}{ Oclusivización } & $/ \mathrm{s} / \rightarrow[\mathrm{d}]$ & $4,55 \%$ & 0 & $2,63 \%$ & 0 & 0 \\
\hline & $/ \mathrm{s} / \rightarrow[\mathrm{t}]$ & $9,09 \%$ & 0 & $34,2 \%$ & 0 & 0 \\
\hline & $/ \mathrm{s} / \rightarrow[\mathrm{ts}]$ & $4,55 \%$ & 0 & $2,63 \%$ & 0 & 0 \\
\hline & Total & $18,19 \%$ & $\mathbf{0}$ & $39,46 \%$ & 0 & 0 \\
\hline \multirow{4}{*}{ Anteriorización } & $/ \mathrm{s} / \rightarrow[\theta]$ & $13,36 \%$ & $14 \%$ & 0 & 0 & $16,22 \%$ \\
\hline & $/ \mathrm{s} / \rightarrow[ð]$ & 0 & 0 & 0 & 0 & 0 \\
\hline & $/ \mathrm{s} / \rightarrow[ð]$ & 0 & 0 & 0 & 0 & 0 \\
\hline & Total & $13,36 \%$ & $14 \%$ & $\mathbf{0}$ & 0 & $16,22 \%$ \\
\hline Posteriorización & $/ \mathrm{s} / \rightarrow\left[\int\right]$ & 0 & $4 \%$ & 0 & 0 & 0 \\
\hline $\begin{array}{l}\text { Oclusivización y } \\
\text { posteriorización }\end{array}$ & $/ \mathrm{s} / \rightarrow[\mathrm{t}]$ & $13,36 \%$ & $2 \%$ & $2,63 \%$ & 0 & 0 \\
\hline Sonorización & $/ \mathrm{s} / \rightarrow[\mathrm{z}]$ & 0 & 0 & 0 & $4,26 \%$ & 0 \\
\hline Omisión & $/ \mathrm{s} / \rightarrow[\mathrm{d}]$ & $45,45 \%$ & $30 \%$ & $57,9 \%$ & $12,77 \%$ & $48,7 \%$ \\
\hline
\end{tabular}

\section{Tabla 2}

Niños de 3 a 4 - Síntesis global de ajustes realizados

\begin{tabular}{|c|c|c|c|c|c|c|}
\hline \multicolumn{2}{|c|}{ Ajustes } & \multicolumn{5}{|c|}{ Niño(a) } \\
\hline Ajuste & Subtipo & 6 & 7 & 8 & 9 & 10 \\
\hline Normal & & $70 \%$ & $6,38 \%$ & $71,15 \%$ & $81,82 \%$ & $71,43 \%$ \\
\hline \multirow{4}{*}{ Oclusivización } & $/ \mathrm{s} / \rightarrow[\mathrm{d}]$ & 0 & 0 & & 0 & 0 \\
\hline & $/ \mathrm{s} / \rightarrow[\mathrm{t}]$ & 0 & & $1,92 \%$ & 0 & $2,38 \%$ \\
\hline & $/ \mathrm{s} / \rightarrow[\mathrm{ts}]$ & 0 & & & 0 & $7,14 \%$ \\
\hline & Total & $\mathbf{0}$ & & $1,92 \%$ & 0 & $9,52 \%$ \\
\hline \multirow{4}{*}{ Anteriorización } & $/ \mathrm{s} / \rightarrow[\theta]$ & 10 & $48,93 \%$ & 0 & $9,09 \%$ & \\
\hline & $/ \mathrm{s} / \rightarrow[ð]$ & 0 & $4,26 \%$ & 0 & 0 & $2,38 \%$ \\
\hline & /s/ $\rightarrow$ [ð] & 0 & 0 & $3,85 \%$ & 0 & \\
\hline & Total & $\begin{array}{l}10 \\
\%\end{array}$ & $53,19 \%$ & $3,85 \%$ & $9,09 \%$ & $2,38 \%$ \\
\hline Posteriorización & $/ \mathrm{s} / \rightarrow\left[\int\right]$ & 0 & 0 & 0 & 0 & 0 \\
\hline $\begin{array}{l}\text { Oclusiv. y } \\
\text { posterioriz }\end{array}$ & $/ \mathrm{s} / \rightarrow[\mathrm{t}]$ & 0 & 0 & $1,92 \%$ & 0 & 0 \\
\hline Sonorización & $/ \mathrm{s} / \rightarrow[\mathrm{z}]$ & 0 & $2,133 \%$ & $15,38 \%$ & 0 & $16,67 \%$ \\
\hline Omisión & /s/ a $\varnothing$ & $20 \%$ & $36,17 \%$ & $5,77 \%$ & $9,09 \%$ & 0 \\
\hline
\end{tabular}


Tabla 3

Sintesis global comparativa de ambos grupos

\begin{tabular}{|c|c|c|c|}
\hline Ajuste & Subtipo & $\begin{array}{c}2-3 \\
\text { años }\end{array}$ & 3-4 años \\
\hline Normal & /s/ & $40,7 \%$ & $55,8 \%$ \\
\hline \multirow[t]{4}{*}{ Oclusivización } & $/ \mathrm{s} / \rightarrow[\mathrm{d}]$ & $1 \%$ & $0 \%$ \\
\hline & $/ \mathrm{s} / \rightarrow[\mathrm{t}]$ & $7,8 \%$ & $1,1 \%$ \\
\hline & $/ \mathrm{s} / \rightarrow[\mathrm{ts}]$ & $1 \%$ & $1,7 \%$ \\
\hline & Total & $9,8 \%$ & $2,7 \%$ \\
\hline \multirow[t]{4}{*}{ Anteriorización } & $/ \mathrm{s} / \rightarrow[\theta]$ & $8,3 \%$ & $14,9 \%$ \\
\hline & $/ \mathrm{s} / \rightarrow[ð]$ & $0 \%$ & $1,2 \%$ \\
\hline & $/ \mathrm{s} / \rightarrow[ð]$ & $0 \%$ & $1,8 \%$ \\
\hline & Total & $8,3 \%$ & $17,8 \%$ \\
\hline Posteriorización & $/ \mathrm{s} / \rightarrow\left[\int\right]$ & $1 \%$ & $0 \%$ \\
\hline $\begin{array}{c}\text { Oclusiv. y } \\
\text { posterioriz }\end{array}$ & $/ \mathrm{s} / \rightarrow[\mathrm{t}]$ & $2,6 \%$ & $0,6 \%$ \\
\hline Sonorización & $/ \mathrm{s} / \rightarrow[\mathrm{z}]$ & $1 \%$ & $8,8 \%$ \\
\hline Omisión & $/ \mathrm{s} / \rightarrow \varnothing$ & $36,6 \%$ & $14,3 \%$ \\
\hline
\end{tabular}

Según la información presentada, puede observarse que el porcentaje de realizaciones "normales" (es decir, sin ningún ajuste) es mayor en los niños de 3 a 4 años por un $15 \%$. Asimismo, las oclusivizaciones a nivel general son menos frecuentes en este grupo, al igual que las posteriorizaciones, las oclusivizaciones-posteriorizaciones y las omisiones.

En lo que respecta a las anteriorizaciones, es notorio que existe un mayor porcentaje de estas realizaciones en el grupo de niños mayores que en el de niños menores. Este dato puede explicarse en parte porque el niño 7, como se observa en la Tabla 2, presenta una abrumadora diferenciación en relación con los otros niños de su grupo etario, ya que tiene un $53 \%$ de realizaciones de /s/ anteriorizada, frente a los demás que las presentan en un rango del $2 \%$ al $10 \%$. Asimismo, en ambos grupos la anteriorización más frecuente fue la que implicaba únicamente el cambio de /s/ a [- estridente], es decir, a [ $\theta]$.

Las sonorizaciones son más frecuentes en el grupo de niños mayores. Una explicación plausible es que esta realización es frecuente en el habla estándar del español de América, como señalan los autores citados Quesada Pacheco (2010) y Calvo (1997), por lo cual algunos de los niños mayores pueden estar presentando ya realizaciones alofónicas más cercanas al habla adulta en lo que respecta a la sonorización de /s/. 
Las omisiones de /s/ son el ajuste más frecuente en el grupo de niños de 2 a 3 años, mientras que en el grupo de 3 a 4 ocurrieron con aproximadamente la mitad de la frecuencia. A continuación, se presenta un análisis de cada ajuste con algunos ejemplos concretos.

\section{- Oclusivización:}

○ /s/ $\rightarrow$ [d]: Ejemplos: [do'pita] sopita, [loe' dobu'kañ] relación con este último ejemplo también podría argumentarse que lo que ocurrió fue una omisión de /s/ seguida de una sonorización de /t/ al quedar en contacto con la vocal). Se presentó solamente en 2 niños de 2 a 3 años, en 2 ocasiones. En una de ellas ocurrió en posición inicial de palabra, es decir, en el ataque silábico.

○ /s/ $\rightarrow$ [t]: Ejemplos: [untapa'tita] un zapatito, [țepe'djo] se perdió, ['ti] sí, ['kata] casa, [di:o:'tawrjo] dinosaurio. Ocurrió siempre en posición de ataque silábico, predominantemente en sílaba tónica, y frecuentemente en posición intervocálica. Fue la más frecuente de los subtipos de oclusivizaciones registrados, y la mayoría de casos ocurrieron en el grupo de 2 a 3 años.

○ /s/ $\rightarrow$ [ts]: Ejemplos: ['tsi] sí, [dețo' loretsu' pelo] de colores su pelo. Ocurrió en dos ocasiones en el grupo de 2 a 3 años. Como se observa, uno ocurrió en la palabra "su" y en combinación con otro fonema /s/, lo cual originó en la pronunciación africada.

\section{- Anteriorización:}

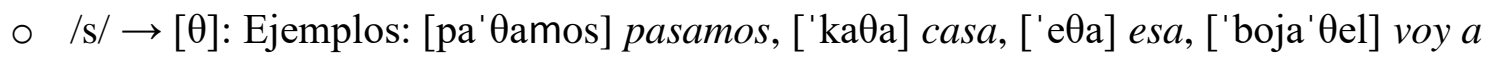

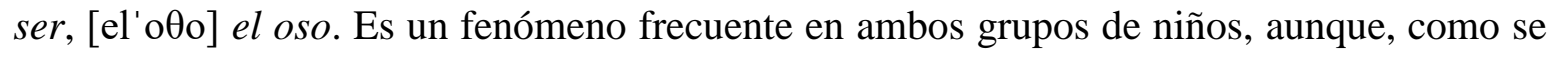
dijo, se presenta en más ocasiones en el grupo de niños mayores. Es la más frecuente de todas las anteriorizaciones, y ocurre mayoritariamente en ataque silábico, y en sílaba tónica o postónica, tanto al inicio como al final de palabra. Suele aparecer en posición intervocálica, o bien antes o después de una vocal.

Puede argumentarse que este fenómeno de anteriorización es "individual" en el sentido de que no es tan generalizado como la omisión, por ejemplo, y de que es propia del habla de cada niño. Es decir, los niños que presentan estas anteriorizaciones las realizan con frecuencia y no de forma aislada, como una característica del habla propia de ese niño. 
Sin embargo, los niños suelen alternar indistintamente entre la variante anteriorizada de /s/ y la "normal", en una misma palabra. Por ejemplo, pueden decir ['ese] y luego ['e日e], para ese. Esto sostiene el argumento de que dicha anteriorización de /s/ no tiene una motivación estrictamente fonológica (a pesar de que la posición intervocálica favorece esta anteriorización) sino que se debe en parte a las diferencias en el proceso de erupción dentaria en los niños, como sostienen Fernández y Cano (2011).

$\circ \quad / \mathrm{s} / \rightarrow[ð]:$ Ejemplos: [jenn'toðes] y entonces, [ðe' pone] se pone, ['tamupe'ðao] está mиy pesado. Solo hubo tres casos registrados de este ajuste, todos en los niños mayores. Como se observa en los ejemplos, este cambio suele ocurrir en ataque silábico, al inicio de palabra, o en posición intervocálica.

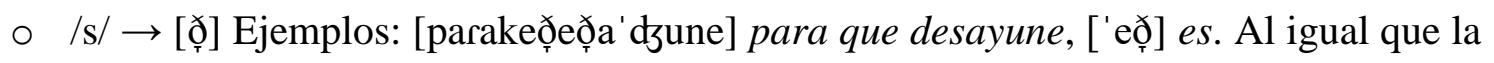
anteriorización pasada, esta ocurre únicamente en tres ocasiones en posición intervocálica.

- Posteriorización: /s/ $\rightarrow$ [J]. Ejemplo: ['e e e 'e: mi'pato] ese es mi pato. Este fenómeno fue bastante infrecuente, ya que solamente se dio en dos ocasiones, en el niño 2, en posición postónica y de ataque silábico. Sin embargo, Fernández y Cano (2011), en su investigación realizada en España sobre procesos fonológicos de niños de 2 a 3 años mencionan que la posteriorización de /s/ fue un fenómeno muy común. Esta discordancia en los resultados puede explicarse diatópicamente.

- Oclusivización y posteriorización: /s/ $\rightarrow$ [t]]. Ejemplo: ['tfi] sí, ['tfinko] cinco, [e'tfa] está. Este ajuste también es relativamente infrecuente; se da en 5 ocasiones en niños de 2 a 3 , y en 1 en niños de 3 a 4. Ocurre mayoritariamente en posición inicial de palabra y en sílaba tónica. El contexto más frecuente de aparición es ante /i/ tónica, y esto es relevante porque la i es una vocal con el rasgo [+ alto], lo cual puede favorecer el ajuste hacia [t]], que también tiene el rasgo [+alto].

- Sonorización: /s/ $\rightarrow$ [z]. Ejemplos: ['eto'ezu'nino] esto es un niño, [dzapa'zo] ya pasó, ['mutfoz nino' ợoros] muchos inodoros, [un'ozo] un oso, [suza'midos] sus amigos. Este fenómeno ocurre más frecuentemente en los niños mayores, lo cual, según se argumentó, puede ser un indicio de que se están adquiriendo los ajustes frecuentes del habla adulta. Este argumento se ve reforzado con el hecho de que el único niño que presenta sonorización 
en el grupo de niños menores es el que tiene un mayor porcentaje de realizaciones normales, y el único ajuste que presenta es el de sonorización.

La sonorización de /s/ se da tanto a inicio como a final de palabra, en sílabas mayoritariamente tónicas o postónicas, e indistintamente en el ataque o en coda silábica. Un contexto frecuente de aparición de este ajuste es en posición intervocálica, o ante una consonante sonora. Uno de los niños alternaba entre variantes sonorizadas y normales de una misma palabra: ['este] ['ezte].

- Omisión: /s/ $\rightarrow \emptyset$. Ejemplos: [mujpeke'jitə] muy pequeñitos, ['ete] este, [bayka'njeß̧е] Blancanieves, [bu'kãnd do'toðָa'pate] buscando todas partes, ['mutfo di:o'tawrjo] muchos dinosaurios, ['guta] gusta. Las omisiones de /s/ ocurrieron mayoritariamente en coda silábica, lo cual concuerda con los hallazgos de Fernández y Cano (2011, p. 77), quienes encontraron que más del $78 \%$ de las omisiones correspondían a la coda silábica. Cerca de la mitad de las omisiones ocurrieron en final de palabra.

Como se mencionó, las omisiones representan el segundo ajuste más común en los niños. Ello lleva a la conclusión de que la omisión de /s/ es un fenómeno generalizado de todo el grupo de niños, que, sin embargo, se reduce considerablemente en los niños mayores.

Es frecuente que ocurra, como se dijo, en final de palabra, en la formación de plurales (os, -as, -es). Resulta notorio que Fernández y Cano (2011, p. 80) hallaron que /s/ estaba “exenta de alteraciones cuando actúa como alternante del morfema plural" y que "se resiste a desaparecer sin dejar rastro en aquellas formas en que desempeña el papel de instrumento gramatical”. En el habla de los niños se registran múltiples omisiones de $\{-$ os/-as $\}$ ya sea en palabras átonas (como "la(s)") o acentuadas como "pequeñito(s)", "parte(s)"). Nótese que en "pequeñito(s)" se evidencia un cambio vocálico debido a la pérdida de la $-\mathrm{s}$ plural. Asimismo, se registra en mayor medida la omisión de /s/ en posición implosiva no necesariamente final (como “e(s)to, e(s)e”). Muchos niños alternan entre la omisión y la realización normal de estos determinantes (como “esto/e(s)to"), lo cual podría indicar, de nuevo, que la motivación no es necesariamente fonológica sino propia del habla de cada niño.

- Normal: Las realizaciones "normales" de /s/ ocurren más frecuentemente en niños mayores de los de 2-3 años, probablemente por razones de edad y mayor adquisición de la variante "estándar" o "adulta”. Sin embargo, no dejan de ser las realizaciones más frecuentes 
en ambos grupos (con $40 \%$ y $55 \%$, de los casos, respectivamente). Se evidencia una marcada tendencia por la pronunciación regular de /s/ en sílabas tónicas y postónicas, en ambos grupos de niños.

\section{Conclusiones y principales focos de reflexión}

A partir de este estudio se pueden efectuar algunas conclusiones que brindan un panorama general de la situación en torno a los ajustes fonético-fonológicos del fonema /s/ en estos niños costarricenses. Algunas ideas que resultan vitales a partir de lo hallado son las siguientes:

- La omisión parece ser un fenómeno más frecuente en los niños menores (de 2 a 3 años); los mayores reducen las omisiones a más de la mitad, en comparación con los menores. Se podría plantear, entonces, que estos resultados están en concordancia con los hallazgos de Soto y Torres (2011), que indican que las omisiones se van reduciendo progresivamente de los 3 a los 6 años, hasta desaparecer casi por completo.

- Resulta pertinente un estudio de índole fonológica y morfológica que examine más a profundidad las omisiones de $\{-\mathbf{s}\}$ cuando es marcador de morfema plural, para determinar si esta omisión es realmente generalizada en el habla infantil costarricense, y si la -s en posición final con función gramatical es un factor que limita la omisión, o bien si esta deja rastros fonéticos (como apertura vocálica ante la omisión), o morfológicos (como la marcación del plural en una palabra pero no en otra).

- La oclusivización y la posteriorización de /s/ son más frecuentes en el habla de niños menores. De la misma forma, se podría plantear que estos ajustes se van perdiendo paulatinamente hasta cerca de los 6 años de edad, aunque Soto y Torres (2011) aducen que las sustituciones de /s/ parecen desaparecer completamente después de los 4 años.

- La sonorización es un alófono frecuente y esperable en el habla adulta estándar del español de Costa Rica, y esto coincide con que fue más frecuente en los niños mayores que en los menores. Lo anterior lleva a argumentar que probablemente estos niños están más cercanos (o acercándose más) a la variante estándar que los menores. 
- Muchos niños alternan libremente entre algún ajuste fonético-fonológico y la producción normal o prototípica del fonema, de lo cual se deduce que muchos de los ajustes que realizados no tienen necesariamente una motivación fonológica, sino que también dependen de otros factores como el desarrollo de la dentición y de las habilidades articulatorias individuales, como argumentan Fernández y Cano (2011). Un ejemplo es el caso expuesto de esto, donde un mismo niño a veces pronuncia ['esto], ['eto] de forma indistinta.

- Ello no quiere decir, por supuesto, que no existan entornos fonológicos proclives para facilitar o promover determinados ajustes, ya que los resultados demuestran que sí los hay: por ejemplo, en el caso de /s/, la posición intervocálica, el final de palabra, la cercanía con otro fonema de rasgos similares, etc. Asimismo, los ajustes de /s/ a [t] (uno de cuyos rasgos es [+alta]), ocurrieron en muchas ocasiones cuando seguía una vocal alta anterior acentuada: $\{1\}$. Otro ejemplo es el caso de muchas sonorizaciones de /s/ que ocurrieron en contacto con una consonante sonora.

- A pesar de que se pueden señalar tendencias generales en los ajustes realizados por los niños, es indudable que el factor individual juega un papel fundamental. Por ejemplo, hay niños que tienden a anteriorizar más, como el niño 7; y otros que tienen una marcada tendencia por la omisión, como el niño 3. El niño 7, a pesar de estar en el grupo etario de 3 a 4 años, tiene un porcentaje de realizaciones normales muy bajo; y a la inversa, el niño 4, que está en el de 2 a 3, tiene un porcentaje alto de realizaciones normales.

- Por lo tanto, el factor individual es relevante para la discusión de los ajustes fonéticofonológicos del habla infantil, ya que el proceso de adquisición y desarrollo fonológico es individual y progresivo. Además, el rango de edad entre los 2 y los 4 años corresponde a una etapa intermedia entre el habla infantil y el desarrollo de la variante estándar de su dialecto. Esta aseveración se ve fortalecida por estudios que abarcan más rangos de edad, como el de Soto y Torres (2011), que evidencian la continuidad del proceso desde los 3 hasta los 6 años.

- Es necesario subrayar que los datos presentados no pretenden establecer generalizaciones para estos grupos etarios, sino exponer los resultados de los diez niños, 
así como algunas de las posibles rutas metodológicas en cuanto a la recolección y análisis de datos para que estos puedan ampliarse, corroborarse y contrastarse en estudios futuros. La aplicación de métodos estadísticos en estudios con poblaciones masivas ayudará a determinar los ajustes fonético fonológicos comunes a nivel poblacional.

- A pesar de la urgencia de seguir incorporando metodologías estadísticas para abarcar una mayor cantidad de población en estas investigaciones, es patente, asimismo, la necesidad y pertinencia de llevar a cabo estudios más focalizados como el presente en el ámbito del análisis de ajustes fonético-fonológicos en el habla infantil. Los estudios individualizados son enriquecedores no solo para explorar inicialmente cada fonema y población estudiada, y así determinar los problemas particulares que exigen, sino también para profundizar en los procesos llevados a cabo por cada niño en estudio, ya que muchos de los ajustes son marcadamente individuales. Es decir, para este ámbito de estudio lingüístico no debe nunca sacrificarse la generalización estadística con la profundización individualizada.

\section{Referencias bibliográficas}

Bosch, L. (1983). El desarrollo fonológico infantil: una prueba para su evaluación. Anuario de psicología, 87-114.

Calvo, A. (1997). El fonema fricativo/s/ en el habla culta de San José: implicaciones teóricas en el sistema fonológico de las coronales. Revista de Filología y Lingüística de la Universidad de Costa Rica, 23(1), 235-246. Recuperado de http://revistas.ucr.ac.cr/index.php/filyling/article/view/20399/20647

Carr, P. (1993). Phonology. Londres: Macmillan.

Fernández, I., \& Cano, P. (2011). Contribución al estudio del desarrollo fonético-fonológico infantil. Procesos fonológicos comunes en niños castellanohablantes de 2 a 4 años. En M. Fernández, Lingüística de corpus y adquisición de lengua. (p. 37-86). Madrid: Arco. 
MacWhinney, B. (2014). Tools for Analyzing Talk. Part 1: The CHAT Transcription Format. Recuperado de The PHILDES Project: http://childes.psy.cmu.edu/manuals/CHAT.pdf

MacWhinney, B. (2014). Tools for Analyzing Talk. Part 2: The CLAN Programs. Recuperado de http://childes.psy.cmu.edu/manuals/CLAN.pdf

Quesada, M. (1996). Los fonemas del español de Costa Rica. Aproximación dialectológica. Lexis, 20(1-2), 535-562.

Quesada, M. (2010). El español de América. San José: Editorial Tecnológica de Costa Rica.

Soto, J., León, H., \& Torres, V. (2011). Una propuesta para la clasificación de los ajustes fonético-fonológicos del habla infantil (CLAFF). Onomázein, 69-79. Recuperado de http://www.onomazein.net/Articulos/23/3_SotoBarba.pdf

Vivar, P., \& León, H. (2009). Desarrollo fonológico-fonético en un un grupo de niños entre 3 y 5,11 años. CEFAC, 190-198.

\section{(c) $(1) \ominus$}

Esta obra está bajo una licencia de Creative Commons Reconocimiento-NoComercial-

SinObraDerivada 4.0 Internacional 